ren um zwei Jahre je Kind ( $\mathbb{S}$ Abs. 1 S. 3 WissZeitVG). Von den grundsätzlich zulässigen sechs Jahren wird in der Praxis regelmäßig kein Gebrauch gemacht - im Einzelfall ist das aber von der jeweiligen Doktormutter oder dem Doktorvater abhängig.

\section{Vereinbarkeit von Beruf und Familie in der Wissenschaft}

Durchaus gewünscht, aber noch nicht geplant, bin ich nach einem Jahr meiner Anstellung schwanger geworden und gehe in Kürze in Mutterschutz. Meine Erfahrungen im Hinblick auf das Mutterwerden in der Wissenschaft sind vermutlich nicht repräsentativ, ich möchte sie aber dennoch weitergeben. Meine Doktormutter hat sehr erfreut und durchweg positiv auf meine Schwangerschaft reagiert, sie hat von Anfang an klargestellt, dass ich keinerlei Nachteile fürchten muss, dass ich jegliche Planungsfreiheit und Flexibilität habe und die durch Mutterschutz und Elternzeit ausfallende Zeit selbstverständlich an den befristeten Arbeitsvertrag angehängt wird. Tatsächlich bietet die Tätigkeit in einer Forschungseinrichtung den Vorteil, dass man jederzeit ersetzbar ist - die Aufgaben werden dann eben durch jemand anderen oder etwas später erledigt. Frau kann auch jederzeit wieder einsteigen, ohne den Anschluss zu verpassen. Zudem stellen die in der Regel flexiblen Arbeitszeiten in der Schwangerschaft eine große Erleichterung dar. Das gilt auch für die Zeit nach der Elternzeit: flexible Arbeitszeiten erleichtern die Kinderbetreuung und Teilzeitlösungen sollten in der Regel möglich sein. Auch Heimarbeitsmöglichkeiten sind denkbar. Ein Problem kann sich bei dem im Wissenschaftsbereich typischen befristeten Arbeitsverträgen ergeben, wenn diese aus Drittmitteln finanziert werden. Grundsätzlich gilt für befristete Arbeitsverhältnisse, dass diese durch Schwangerschaft oder Elternzeit nicht etwa automatisch verlängert werden, sondern zum vereinbarten Zeitpunkt einfach auslaufen. Bei Befristungen nach dem WissZeitVG verlängert sich die Dauer eines befristeten Arbeitsvertrages hingegen im Einverständnis (dieses ist gegenüber der Arbeitgeberin bzw. dem Arbeitgeber zu erklären) mit der wissenschaftlichen Mitarbeiterin oder dem wissenschaftlichen Mitarbeiter um Zeiten der Inanspruchnahme von Elternzeit und um Zeiten des Beschäftigungsverbotes im Rahmen des Mutterschutzes (vgl. $\mathbb{S}$ Abs. 5 S. 1 Nr. 3 WissZeitVG), soweit in diesen Zeiträumen keine Erwerbstätigkeit erfolgt ist. Zudem wird die Verlängerung nicht auf die oben erwähnte Höchstbefristungsdauer von grundsätzlich sechs Jahren angerechnet ( $\mathbb{} 2$ Abs. 5 S. 2 WissZeitVG). Eine Ausnahme davon gilt wiederum für wissenschaftliche Mitarbeiterinnen und Mitarbeiter, die überwiegend aus Drittmitteln finanziert werden, $\mathbb{} 2$ Absatz 2 WissZeitVG: $\mathbb{} 2$ Absatz 5 WissZeitVG greift für sie nicht, so dass sich ihre Arbeitsverträge nicht verlängern. Eine Verlängerung kommt dann nur in Betracht, wenn die Drittmittelgeberin oder der Drittmittelgeber entsprechende Personalmittel zur Verfügung stellt. Im Übrigen ist die Frage der Vereinbarkeit stark von der oder dem jeweiligen Vorgesetzten abhängig. Dennoch denke ich, dass Forschungseinrichtungen und Hochschulen eher aufgeklärte und positive Rahmenbedingungen für Frauen mit sich bringen.

Negativer waren zum Teil einige Reaktionen im weiteren kollegialen Umfeld und Bekanntenkreis. Die häufigste Frage war wohl: „Und deine Promotion?!“ Für viele Promovierende scheint es undenkbar zu sein, während der Promotion ein Kind zu bekommen. Manch eine fühlt sich durch eine solche Entscheidung vielleicht auch selbst in Frage gestellt, lautet doch das eigene Credo „Kind oder Karriere“. Ich persönlich bin mittlerweile froh, unter diesen privilegierten Umständen ein Kind bekommen zu können. In meiner derzeitigen Lage habe ich sicher mehr Freiheiten und Flexibilität als in anderen Berufen. Sicher kann es sein, dass die Promotion langsamer vorangeht, vielleicht ist aber auch das Gegenteil der Fall. Planbar ist das vorher nicht, aber wie ein bekanntes djb-Mitglied immer sagt: „Verabschieden Sie sich von der Idee der Planbarkeit!“

\title{
Ja, es geht! - Ein Erfahrungsbericht zum Examen ohne Repetitor
}

\author{
Anne Brozat, LL.M. (UWC) \\ Promotionsstipendiatin, Ruhr-Universität Bochum
}

Spätestens während des Schwerpunktstudiums kommt bei jeder Jurastudentin die Frage auf: „Zu welcher Repetitorin oder zu welchem Repetitor soll ich gehen? “ In dieser Zeit hört man sich um und vielleicht hört man auch von Kommilitoninnen oder Kommilitonen, die sich ohne Repetitorin oder Repetitor auf das Examen vorbereiten bzw. vorbereitet haben. Ich selbst kannte im Oktober 2007, als ich die mündliche Prüfung des Schwerpunktbereichs gerade hinter mir hatte, niemanden, der sich ohne Repetitorium vorbereiten wollte. Deswegen war auch mein erster Gedanke nicht: „Soll ich „Rep“ machen oder mich alleine vorbereiten?“, sondern: „Zu welcher oder welchem „Rep“ soll ich gehen?“ Gleichzeitig begann ich zu überlegen, wann ich Examen schreiben wollte. Für meinen Freiversuch hatte ich noch bis Oktober 2008 Zeit; allerdings war ich mir nicht sicher, ob ich ein Jahr lang auch tatsächlich kontinuierlich lernen würde. So kam mir, auch durch Gespräche mit meinen Eltern, die Idee, bereits im Frühjahr 2008 den Freiversuch anzugehen. In Berlin gibt es pro Jahr zwei Termine für das erste Examen (einen im Frühjahr und einen im Herbst) und Abschichten ist nicht möglich. 


\section{Die Vorüberlegungen}

Ich hatte also ca. sechs Monate Zeit, um den gesamten examensrelevanten Stoff noch einmal zu wiederholen. Da aber alle Repetitorien auf einen Zeitraum von einem Jahr ausgelegt sind, fiel das „Rep“ als Option auf einmal weg.

Deswegen ging ich dazu über, mir zu überlegen, wie ich mich allein vorbereiten könnte. Dazu druckte ich mir als erstes die entsprechende Stelle der Ausbildungsordnung aus. Dort ist jedes Gebiet aufgeführt und festgeschrieben, ob vertieftes Wissen verlangt wird oder lediglich Grundzüge. Als zweiten Schritt lieh ich mir Lehrbücher aus, um einen Überblick über den Stoff zu bekommen und begann einen Lernplan zu erstellen, in dem ich die Gebiete, in denen Grundwissen verlangt wird, vorerst ausklammerte. Bei der Vorbereitung haben mir auch Homepages von Personen geholfen, die ihr Examen selbst ohne "Rep“ geschrieben haben, insbesondere die von Peter Finke. ${ }^{1}$

\section{Das Lernen}

Am Anfang versuchte ich den Stoff mit Hilfe von Lehrbüchern zu erarbeiten. Mir waren die Lehrbücher aber teilweise viel zu wenig am Fall ausgerichtet, deswegen habe ich mich bald auf die Bücher aus dem Verlag Dr. Rolf Schmidt, dem Fall-Fallag und die kleinen Bücher aus der Hemmer-Reihe „Die ... wichtigsten Fälle zum ..." beschränkt. Ich wollte mir die Grundlagen genau erarbeiten und alles aus den ersten Semestern wirklich verstehen und nicht nur auswendig lernen. $\mathrm{Zu}$ diesem Zweck hatte ich immer ein Gesetz neben mir liegen, in das ich mir auch Kommentierungen geschrieben und Zusammenhänge notiert habe. Auch wenn ich dieses Gesetz im Examen nicht benutzen konnte, weil in Berlin Kommentierungen nicht erlaubt sind, so hat es mir beim Lernen geholfen, die Systematik zu verstehen und zu erkennen, was ich tatsächlich auswendig lernen muss und was im Gesetz steht. Im Strafrecht habe ich als „Lehrbuch“ den Studienkommentar von Joecks verwendet, der sich auf das Wesentliche beschränkt und auf die Examensrelevanz der einzelnen Normen mit Hilfe kleiner Sternchen hinweist. Konkret sah es so aus, dass ich ein Stoffgebiet mit dem Lösen der Minifälle aus dem Fall-Fallag zu erarbeiten begonnen habe. Dann habe ich nachgelesen, was mir unklar war und bei Verständnisproblemen mit einem anderen Lehrbuch noch vertieft. Danach habe ich mir die Fälle von Hemmer vorgenommen, wobei ich die Lösungsseite immer mit einem Blatt abgedeckt habe. Die Lösungen habe ich nicht immer ausformuliert, aber Standardformeln, wie sie in jeder Klausur vorkommen, habe ich ausgeschrieben, um sie im Examen ohne großes Nachdenken aufschreiben zu können. Dabei habe ich, außer für Definitionen im Strafrecht, keine extra Karteikarten angefertigt; die Gesetzestexte, in denen ich selbst Notizen gemacht habe, waren sozusagen meine Karteikarten.

\section{Das Uni-Repetitorium}

Im ersten Monat bin ich probehalber auch zum Uni-Rep der Freien Universität gegangen. Allerdings hat mir das Uni-Rep nicht so sehr zugesagt. Es ist auch auf ein Jahr angelegt und mir war der Stoff teilweise zu unübersichtlich aufbereitet. Ich muss auch zugeben, dass mich in dieser Vorbereitungsphase einige Kommilitoninnen und Kommilitonen gestört haben. Manche präsentieren nur zu gern Gelerntes und meinen, genau zu wissen, was besonders wichtig sei und was man ganz bestimmt wissen müsse. Diesem Gerede war ich bei meiner eigenen Vorbereitung zu Hause nicht ausgesetzt. Nun habe ich aber auch das Glück, einen Juristen als Vater zu haben, der mit mir gerne einzelne Probleme reflektiert und geholfen hat, hartnäckige Verständnisschwierigkeiten zu lösen. Deswegen empfehle ich, wenn man keine Lern-AG hat, sich doch mindestens eine verlässliche Gesprächspartnerin oder einen verlässlichen Gesprächspartner für juristische Fragen in dieser Zeit zu suchen.

\section{Die Übungsklausuren}

Im November - nach einem Monat Lernen - habe ich angefangen, Übungsklausuren an der Uni zu schreiben. Zwar habe ich in diesen Klausuren anfangs miserable Zensuren bekommen - aber egal - der Übungseffekt hat für mich gezählt. Ich habe etwas über Systematik und Aufbau der Examensklausuren gelernt. In den ersten Klausuren habe ich mir beim Lösen auch noch Lehrbücher zu Hilfe genommen. Bald waren meine Wissenslücken aber nicht mehr so groß, so dass ich mich dazu gezwungen habe, nur mit dem Gesetz an der Klausur zu arbeiten. Ab Dezember habe ich pro Woche zwei Klausuren geschrieben, eine an der Humboldt-Universität und eine an der Freien Universität. Bis zum Examen habe ich ungefähr 30 Klausuren geschrieben. Durch die Übungsklausuren hatte ich bei Examensbeginn das Gefühl, mich würde kein Klausurtyp mehr überraschen. Dass mich die drei Zivilrechtsklausuren im Examen dann allein aufgrund der Art der Aufgabenstellung und Fragen doch jedes Mal überrascht haben, war nicht so schlimm, da ich durch die Übungsklausuren ein Muster hatte, wie ich Unbekanntes lösen kann.

\section{Und dann ...}

Ich war mit dem Ergebnis meines Freiversuchs so zufrieden, dass ich nicht noch einmal in den Verbesserungsversuch und stattdessen lieber für ein Masterstudium nach Südafrika gegangen bin. Ich kann nur empfehlen, sich wenigstens mit dem Gedanken zu beschäftigen, das Examen ohne „Rep“ zu machen. Wie man sich vorbereitet, ist auch stark typabhängig, jede lernt anders und es gibt nicht die eine Lösung. Ich möchte mit meinen Erfahrungen aber Mut machen, es ohne „Rep“ zu wagen.

1 〈http://www.examen-ohne-rep.piranho.de> (Zugriff: 27.6.11). 